\title{
617.
}

\section{ON THE SCALENE TRANSFORMATION OF A PLANE CURVE.}

[From the Quarterly Journal of Pure and Applied Mathematics, vol. XIII. (1875), pp. 321-328.]

THE transformation by reciprocal radius vectors can be effected mechanically by Sylvester's Peaucellier-cell. But, employing a more general cell (considered incidentally by him) which may be called the scalene-cell, we have the scalene transformation in question*; viz. if, in two curves, $r, r^{\prime}$ are radius vectors belonging to the same angle (or say opposite angles) $\theta$, then the relation between $r, r^{\prime}$ is

$$
r r^{\prime}\left(r+r^{\prime}\right)+\left(m^{2}-l^{2}\right) r+\left(m^{2}-n^{2}\right) r^{\prime}=0 ;
$$

or, as this may also be written,

$$
r^{2}+\left(r^{\prime}-\frac{l^{2}-m^{2}}{r^{\prime}}\right) r+m^{2}-n^{2}=0
$$

The transformation is, it will be seen, an interesting one for its own sake, independently of the remarkably simple mechanical construction, viz. the scalene cell is simply a system of 3 pairs of equal rods $P A, Q A ; P B, Q B ; P C, Q C$ (fig. 1, p. 528), jointed together at and capable of rotating about the points $P, Q, A, B, C$; the three lengths $P A, P B, P C$ (say these are $=l, m, n$ ) are all of them unequal: in the case of any two of them equal, we have Peaucellier or isosceles cell. The effect of the arrangement is that the points $A, B, C$ are retained in a right line, the distances $B A,=r^{\prime}$, and $B C,=r$, being connected by the above-mentioned equation; so that taking $B$ as a fixed point, if the point $A$ describe any given curve, the point $C$ will describe the corresponding or transformed curve.

In the case where the given curve is a right line or a circle, we may through $B$ draw at right angles to the curve the axis $x^{\prime} B x$ : viz. in the case of the circle,

* The transformation itself, and doubtless many of the results obtained by means of it, are familiar to Prof. Sylvester; and I abandon all elaim to priority. 
the axis $x^{\prime} B x$ passes through its centre; and we measure the angle $\theta$ from this line, viz. we write $\angle x B C=\angle x^{\prime} B A=\theta$.

Fig. 1.

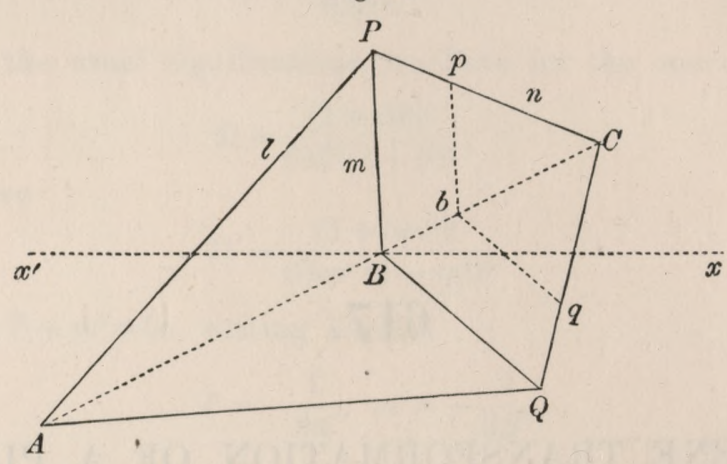

Suppose, first, that the locus of $A$ is a right line, or a circle passing through $B$. Its equation is $r^{\prime}=\frac{c}{\cos \theta}$ or $=c \cos \theta$; and we accordingly have for the transformed curve

$$
r^{2}+\left(\frac{c}{\cos \theta}-\frac{l^{2}-m^{2}}{c \cos \theta}\right) r+m^{2}-n^{2}=0
$$

or else

$$
r^{2}+\left(c \cos \theta-\frac{l^{2}-m^{2}}{c \cos \theta}\right) r+m^{2}-n^{2}=0
$$

viz. multiplying in each case by $r \cos \theta$, and then writing $r \cos \theta=x, r^{2}=x^{2}+y^{2}$, the equations become

$$
x\left(x^{2}+y^{2}\right)+c\left(x^{2}+y^{2}\right)-\frac{l^{2}-m^{2}}{c} a^{2} \quad+\left(m^{2}-n^{2}\right) x=0
$$

and

$$
x\left(x^{2}+y^{2}\right)+c x^{2} \quad-\frac{l^{2}-m^{2}}{c}\left(x^{2}+y^{2}\right)+\left(m^{2}-n^{2}\right) x=0
$$

viz. in each case the curve is a circular cubic passing through the origin $B$ and having an asymptote parallel to the axis of $y$. The curve is nodal, if $m=n$, viz. in this case the origin is a node: or if $c=\sqrt{ }\left(l^{2}-n^{2}\right)+\sqrt{ }\left(m^{2}-n^{2}\right)$.

Suppose next that the locus of $A$ is a circle, centre at a distance $=\gamma$ along $B x^{\prime}$ and radius $=h$ : we have

$$
r^{\prime 2}-2 \gamma r^{\prime} \cos \theta+\gamma^{2}-h^{2}=0
$$

viz. if

or, what is the same thing,

$$
\gamma^{2}-h^{2}=-\left(l^{2}-m^{2}\right)
$$

$$
h^{2}+m^{2}=\gamma^{2}+l^{2}
$$

then we have

$$
r^{\prime}-\frac{l^{2}-m^{2}}{r^{\prime}}=2 \gamma \cos \theta
$$


and the transformed curve is

or, as this may be written,

$$
r^{2}+2 \gamma r \cos \theta+m^{2}-n^{2}=0
$$

$$
r^{2}+2 \gamma r \cos \theta+\gamma^{2}-f^{2}=0
$$

where $\gamma^{2}-f^{2}=m^{2}-n^{2}$, that is, $f^{2}+m^{2}=\gamma^{2}+n^{2}$; viz. this is a concentric circle radius $f$.

The theorem may be presented as follows. Consider two concentric circles, centre $O$ and radii $h, f$ respectively; take an arbitrary point $B$, distance $O B=\gamma$; and taking $m$ arbitrary, determine $l, n$ by the equations

$$
l^{2}=m^{2}+h^{2}-\gamma^{2}, n^{2}=m^{2}+f^{2}-\gamma^{2} ;
$$

then drawing through $B$ an arbitrary line to meet the circles in $A, C$ respectively; also describing a circle, centre $B$ and radius $=m$; and through $O$ drawing a line perpendicular to $A B C$ to meet the last-mentioned circle in two points $P, Q$ : for these points, the distances from the points $A, B, C$ are $=l, m, n$ respectively.

To verify this, take $O$ as the origin, $O B$ for the axis of $x, \theta$ the inclination of $A B C$ to this axis, $B A=r^{\prime}, B C=r$; the coordinates of $C, B, A$ are

$$
\begin{array}{ll}
\gamma+r \cos \theta, & r \sin \theta \\
\gamma \quad & 0 \\
\gamma+r^{\prime} \cos \theta, & -r^{\prime} \sin \theta
\end{array}
$$

whence, taking $(x, y)$ for the coordinates of $P$ (or $Q$ ), the equations to be verified are

$$
\begin{aligned}
(x-\gamma-r \cos \theta)^{2}+(y-r \sin \theta)^{2} & =n^{2}, \\
(x-\gamma)^{2}+y^{2} & =m^{2}, \\
\left(x-\gamma+r^{\prime} \cos \theta\right)^{2}+\left(y+r^{\prime} \sin \theta\right)^{2} & =l^{2} .
\end{aligned}
$$

By means of the second equation, the other two become

$$
\begin{aligned}
-2(x-\gamma) r \cos \theta-2 y r \sin \theta+r^{2}=n^{2}-m^{2}, \\
2(x-\gamma) r^{\prime} \cos \theta+2 y r^{\prime} \sin \theta+r^{\prime 2}=l^{2}-m^{2} ;
\end{aligned}
$$

or, substituting for $n^{2}-m^{2}, l^{2}-m^{2}$ the values $f^{2}-\gamma^{2}$ and $h^{2}-\gamma^{2}$, the equations are

$$
\begin{gathered}
-2 x r \cos \theta-2 y r \sin \theta+r^{2}+2 \gamma r \cos \theta+\gamma^{2}-f^{2}=0, \\
2 x r^{\prime} \cos \theta+2 y r^{\prime} \sin \theta+r^{\prime 2}-2 \gamma r^{\prime} \cos \theta+\gamma^{2}-h^{2}=0,
\end{gathered}
$$

viz. in virtue of the equations of the two circles, these reduce themselves each of them to

$$
x \cos \theta+y \sin \theta=0,
$$

which equation, together with the second equation

$$
(x-\gamma)^{2}+y^{2}=m^{2},
$$

determine $(x, y)$ as above.

C. IX. 
Reverting to the case where the locus of $A$ is the circle

this gives

$$
r^{\prime 2}-2 \gamma r^{\prime} \cos \theta+\gamma^{2}-h^{2}=0
$$

$$
\begin{aligned}
& r^{\prime}=\gamma \cos \theta+\sqrt{ }\left(h^{2}-\gamma^{2} \sin ^{2} \theta\right) \\
& \frac{1}{r^{\prime}}=\frac{\gamma \cos \theta-\sqrt{ }\left(h^{2}-\gamma^{2} \sin ^{2} \theta\right)}{\gamma^{2}-h^{2}}
\end{aligned}
$$

so that for the transformed curve we have

$$
r^{2}+r\left(1-\frac{l^{2}-m^{2}}{\gamma^{2}-h^{2}}\right) \gamma \cos \theta+r\left(1+\frac{l^{2}-m^{2}}{\gamma^{2}-h^{2}}\right) \sqrt{ }\left(h^{2}-\gamma^{2} \sin ^{2} \theta\right)+m^{2}-n^{2}=0
$$

Putting for shortness $\frac{l^{2}-m^{2}}{\gamma^{2}-h^{2}}=\lambda$, and for $r, r \cos \theta, r \sin \theta$, writing $\sqrt{ }\left(x^{2}+y^{2}\right), x, y$ respectively, this is

$$
x^{2}+y^{2}+(1-\lambda) \gamma x+(1+\lambda) \sqrt{ }\left\{h^{2}\left(x^{2}+y^{2}\right)-\gamma^{2} y^{2}\right\}+m^{2}-n^{2}=0,
$$

or, what is the same thing,

$$
\left\{x^{2}+y^{2}+(1-\lambda) \gamma x+m^{2}-n^{2}\right\}^{2}=(1+\lambda)^{2}\left\{h^{2}\left(x^{2}+y^{2}\right)-\gamma^{2} y^{2}\right\}
$$

a bicircular quartic. In the case $\lambda=-1$, it reduces itself to the circle

$$
x^{2}+y^{2}+2 \gamma x+m^{2}-n^{2}=0
$$

twice, which is the case considered above; and in the case $\lambda=1$, or $l^{2}+h^{2}=m^{2}+\gamma^{2}$, the equation is

$$
\left(x^{2}+y^{2}+m^{2}-n^{2}\right)^{2}=4\left\{h^{2}\left(x^{2}+y^{2}\right)-\gamma^{2} y^{2}\right\},
$$

so that the curve is symmetrical in regard to each axis. In the case $\gamma=0$, the locus is a pair of concentric circles, centre $B$.

The equation

$$
\left\{x^{2}+y^{2}+(1-\lambda) \gamma x+m^{2}-n^{2}\right\}^{2}=(1+\lambda)^{2}\left\{h^{2}\left(x^{2}+y^{2}\right)-\gamma^{2} y^{2}\right\}
$$

which contains the four constants $\lambda, \gamma, h$ and $m^{2}-n^{2}$, may be written in the form

$$
\left(x^{2}+y^{2}+A x+B\right)^{2}=a x^{2}+e y^{2},
$$

(where the constants $A, B, a, e$ are also arbitrary). This is, in fact, the equation of the general symmetrical bicircular quartic, referred to a properly-selected point on the axis as origin, viz. the origin is the centre of any one of the three involutions formed by the vertices (or points on the axis); say it is any one of the three involutioncentres of the curve.

To show this, assume

$$
(x-\alpha)(x-\beta)(x-\gamma)(x-\delta)=x^{4}-p x^{3}+q x^{2}-r x+s:
$$


then, taking $B$ arbitrary, the equation of the symmetrical bicircular quartic having for vertices the points $x=\alpha, x=\beta, x=\gamma, x=\delta$, is

$$
\left(x^{2}+y^{2}-\frac{1}{2} p x+B\right)^{2}=\left(2 B+\frac{1}{4} p^{2}-q\right) x^{2}+(r-p B) x+\left(-s+B^{2}\right)
$$

in fact, this is the form of the general equation, and writing therein $y=0$, it becomes $x^{4}-p x^{3}+q x^{2}-r x+s=0$, that is, $(x-\alpha)(x-\beta)(x-\gamma)(x-\delta)=0$. Hence, writing for convenience

the equation is

$$
\begin{aligned}
& A=-\frac{1}{2} p, \\
& a=2 B+\frac{1}{4} p^{2}-q, \\
& b=r-p B \\
& c=-s+B^{2},
\end{aligned}
$$

$$
\left(x^{2}+y^{2}+A x+B\right)^{2}=a x^{2}+b x+c .
$$

This may be written

$$
\left(x^{2}+y^{2}+A x+B+\theta\right)^{2}=(a+2 \theta) x^{2}+2 \theta y^{2}+(b+2 \theta A) x+c+2 B \theta+\theta^{2},
$$

viz. assuming $\theta=-\frac{b}{2 A}$ in order on the right-hand side to destroy the term in $x$, the equation is

$$
\left(x^{2}+y^{2}+A x+B-\frac{b}{2 A}\right)^{2}=\left(a-\frac{b}{A}\right) x^{2}-\frac{b}{A} y^{2}+\frac{1}{4 A^{2}}\left(b^{2}-4 A B b+4 A^{2} c\right),
$$

which is of the form

$$
\left(x^{2}+y^{2}+A x+B\right)^{2}=a x^{2}+e y^{2}+f ;
$$

and if $f=0$, that is, if $b^{2}-4 A B b+4 A^{2} c=0$, then it is of the required form

$$
\left(x^{2}+y^{2}+A x+B\right)^{2}=a x^{2}+e y^{2} .
$$

We have

$$
\begin{aligned}
b^{2}-4 A B b+4 A^{2} c & =(r-p B)^{2}+2 p B(r-p B)+p^{2}\left(-s+B^{2}\right) \\
& =r^{2}-p^{2} s
\end{aligned}
$$

or the required condition is $r^{2}-p^{2} s=0$. But we have

$$
p^{2} s-r^{2}=(\alpha \delta-\beta \gamma)(\beta \delta-\gamma \alpha)(\gamma \delta-\alpha \beta)
$$

as is easily verified by writing

$$
p=\delta+p_{0}, \quad q=\delta p_{0}+q_{0}, \quad r=\delta q_{0}+r_{0}, \quad s=\delta r_{0},
$$

where $p_{0}, q_{0}, r_{0}$ stand for

$$
\alpha+\beta+\gamma, \quad \beta \gamma+\gamma \alpha+\alpha \beta, \quad \alpha \beta \gamma,
$$


respectively. The required condition thus is

$$
(\alpha \delta-\beta \gamma)(\beta \delta-\gamma \alpha)(\gamma \delta-\alpha \beta)=0,
$$

viz. the origin (that is, the fixed point $B$ of the cell) must be at one of the three involution-centres.

Comparing the equation

with the equation

$$
\left\{x^{2}+y^{2}+(1-\lambda) \gamma x+m^{2}-n^{2}\right\}^{2}=(1+\lambda)^{2}\left\{h^{2}\left(x^{2}+y^{2}\right)-\gamma^{2} y^{2}\right\}
$$

we have

$$
\left\{x^{2}+y^{2}+A x+B\right\}^{2}=a x^{2}+e y^{2}
$$

$$
\begin{aligned}
& A=(1-\lambda) \gamma, \\
& B=m^{2}-n^{2}, \\
& a=(1+\lambda)^{2} h^{2}, \\
& e=(1+\lambda)^{2}\left(h^{2}-\gamma^{2}\right),
\end{aligned}
$$

and thence $a-e=(1+\lambda)^{2} \gamma^{2}$. Consequently $\frac{a-e}{A^{2}}=\left(\frac{1+\lambda}{1-\lambda}\right)^{2}$, which gives $\lambda$ : and then $h^{2}=\frac{a}{(1+\lambda)^{2}}, \gamma^{2}=\frac{a-e}{(1+\lambda)^{2}}, m^{2}-n^{2}=B ;$ viz. we thus have the values of $\lambda, h, \gamma$ and $m^{2}-n^{2}$ for the description of a given curve $\left(x^{2}+y^{2}+A x+B\right)^{2}=a x^{2}+e y^{2}$. In order that the description may be possible, $a$ and $a-e$ must be each of them positive.

For the Cartesian $a$ is $=e$, whence $1+\lambda=0$, and the equation becomes

$$
\left(x^{2}+y^{2}+2 \gamma x+m^{2}-n^{2}\right)^{2}=0,
$$

which is a twice repeated circle; hence the Cartesian cannot be constructed by means of a cell as above.

To obtain a construction of the Cartesian, it may be remarked that, if a symmetrical bicircular quartic be inverted in regard to an axial focus, viz. if the focus be taken as the centre of inversion, we obtain a Cartesian. The axial foci of the curve

$$
\left(x^{2}+y^{2}+A x+B\right)^{2}=a x^{2}+e y^{2}
$$

are points on the axis, the abscissa $x=\theta$ being determined by the equation

$$
e\left(\theta^{2}+A \theta+B\right)^{2}-a\left(\theta^{2}-B\right)^{2}-a e \theta^{2}=0 .
$$

The equation referred to a focus as origin is therefore

$$
\left\{x^{2}+y^{2}+(A+2 \theta) x+B+\theta^{2}\right\}^{2}=a x^{2}+e y^{2}+2 a \theta x+\theta^{2} ;
$$

then inverting, viz. for $x, y$ writing $\frac{k^{2} x}{r^{2}}, \frac{k^{2} y}{r^{2}}$ ( $k$ arbitrary), we have, as may be verified the equation of a Cartesian. 
The inversion can be performed mechanically by an ordinary Peaucellier-cell; the complete apparatus for the construction of a Cartesian is therefore as in fig. 2, viz. we have a cell $B A C$ as before, $B$ a fixed point, locus of $A$ a circle (for convenience of drawing, the arrangement has been made $B A C$ instead of $A B C$ ), and we connect with $C$ a Peaucellier-cell $C A^{\prime} B^{\prime}$, arms $n^{\prime}, n^{\prime}, m^{\prime}$, the fixed point $B^{\prime}$ being on the axis, which is the line joining $B$ with the centre of the circle described by $A$. This being so, then $A$ describing a circle, $C$ will describe a symmetrical bicircular quartic, and $A^{\prime}$ will describe the inverse of this, being in general a like curve; but if the position of $B^{\prime}$ be properly determined, viz. if $B^{\prime}$ be at a focus of the first-mentioned quartic,

Fig. 2.

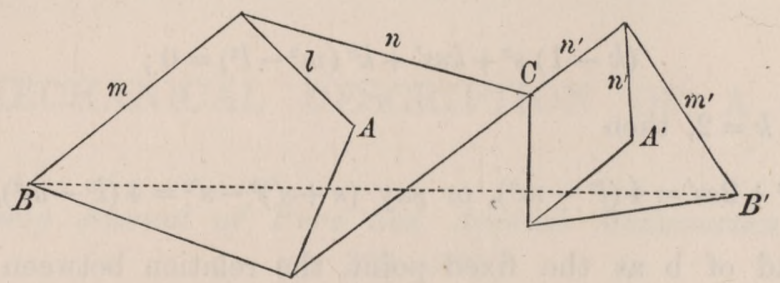

then $A^{\prime}$ will describe a Cartesian. A further investigation would be necessary in order to determine how to adapt the apparatus to the description of a given Cartesian.

A more convenient mechanical description of a Cartesian is, however, that given in the paper which follows the present one [618].

The equation

$$
\left\{x^{2}+y^{2}+(1-\lambda) \gamma x+m^{2}-n^{2}\right\}^{2}=(1+\lambda)^{2}\left\{h^{2}\left(x^{2}+y^{2}\right)-\gamma^{2} y^{2}\right\}
$$

may also be written

$$
\begin{aligned}
& \left\{x^{2}+y^{2}+(1-\lambda) \gamma x-\frac{1}{2}(1+\lambda)^{2}\left(h^{2}-\gamma^{2}\right)+m^{2}-n^{2}\right\}^{2} \\
& \quad=(1+\lambda)^{2}\left\{\gamma^{2} x^{2}-(1-\lambda)\left(h^{2}-\gamma^{2}\right) \gamma x+\frac{1}{4}(1+\lambda)^{2}\left(h^{2}-\gamma^{2}\right)^{2}-\left(m^{2}-n^{2}\right)\left(h^{2}-\gamma^{2}\right)\right\}
\end{aligned}
$$

viz. the equation is now brought into the form

$$
\left(x^{2}+y^{2}+A x+B\right)^{2}=a x^{2}+b x+c .
$$

Expressing the coefficients $A, B, a, b, c$ in terms of $\lambda, \gamma, h, m^{2}-n^{2}$, it appears by what precedes, that we should have identically $b^{2}-4 A B b+4 A^{2} c=0$, viz. this is the equation which expresses that the origin is an involution-centre.

If, instead of the original cell, we consider a new cell obtained by substituting for the arms $P B, B Q$, the arms $\mathrm{pb}, \mathrm{bq}$, jointed on to the points $\mathrm{p}, \mathrm{q}$ on the arms $C P, C Q$ respectively, and instead of $B$, making $b$ the fixed point; then writing $C p=k n$, $\mathrm{pb}=k m$, so that the parameters of the cell are $l, m, n, k$, and taking $C \mathrm{~b}=s, \mathrm{~b} A=s^{\prime}$, 
we have $s=k r, s+s^{\prime}=r+r^{\prime}$, that is, $r=\frac{s}{k}, r^{\prime}=\frac{k-1}{k} s+s^{\prime}$. Substituting in the equation between $r, r^{\prime}$, written for greater convenience in the form

$$
\left(r+r^{\prime}\right)\left(r r^{\prime}+m^{2}-l^{2}\right)+\left(l^{2}-n^{2}\right) r^{\prime}=0,
$$

the relation between $s, s^{\prime}$ is found to be

$$
\left(s+s^{\prime}\right)\left(\frac{k-1}{k^{2}} s^{2}+\frac{s s^{\prime}}{k}+m^{2}-l^{2}\right)+\left(l^{2}-n^{2}\right)\left(\frac{k-1}{k} s+s^{\prime}\right)=0 .
$$

On account of the term in $s^{3}$, this equation in its general form does not, it would appear, give rise to transformations of much elegance. If, however, $l=n$, then the relation becomes

$$
(k-1) s^{2}+k s s^{\prime}+k^{2}\left(m^{2}-l^{2}\right)=0 ;
$$

and in particular, if $k=2$, then

$$
s^{2}+2 s s^{\prime}=4\left(l^{2}-m^{2}\right) \text {, or say }\left(s+s^{\prime}\right)^{2}-s^{\prime 2}=4\left(l^{2}-m^{2}\right),
$$

viz. taking $A$ instead of $\mathrm{b}$ as the fixed point, the relation between the radii $A C, A \mathrm{~b}$ is $\rho^{2}-\rho^{\prime 2}=4\left(l^{2}-m^{2}\right)$; the cell is in this case Sylvester's "quadratic-binomial extractor." 